\title{
GAIA Neonatal Encephalopathy Level of Diagnostic Certainty Terminology
}

National Cancer Institute

\section{Source}

National Cancer Institute. GAIA Neonatal Encephalopathy Level of Diagnostic Certainty

Terminology. NCl Thesaurus. Code C128699.

A subset of terminology related to neonatal encephalopathy, developed by the Global Alignment of Immunization safety Assessment in pregnancy consortium to aid in monitoring and improving fetal and maternal outcomes. 